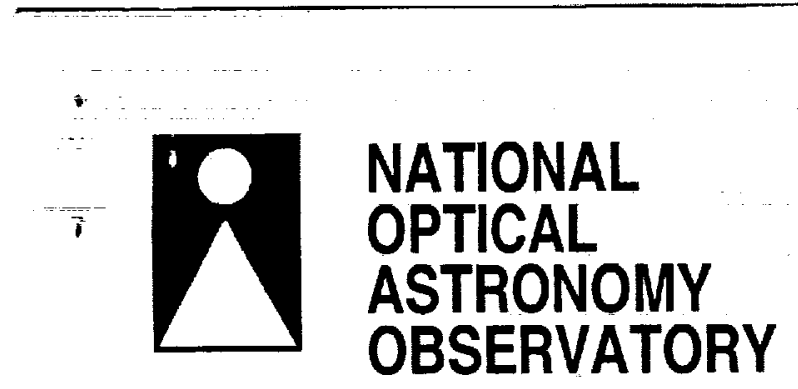

Preprint Series

NOAO Preprint No. 890

A MODEL FOR THE BREAKUP OF COMET LINEAR (C/1999 S4)

Nalin H. Samarasinha

(National Optical Astronomy Observatory)

Accepted by: Icarus

June 2001 

abstract

We propose a mechanism based on the rubble-pile hypothesis of the cometary nucleus (Weissman 1986) to explain the catastrophic breakup of comet LINEAR (C/1999 S4) observed during July-August 2000. We suggest that a solid nucleus made up of 10-100 m "cometesimals" (Weidenschilling 1997) contains a network of inter-connected voids in the inter-cometesimal regions. The production of super-volatile (i.e., species more volatile than water) gasses into these voids occurs due to the thermal wave propagating through the nucleus and associated phase transitions of water ice. The network of voids provides an efficient pathway for rapid propagation of these gasses within the nucleus resulting in gas pressure caused stresses over a wide regime of the nucleus. This provides a mechanism for catastrophic breakups of small cometary nuclei such as comet LINEAR (C/1999 S4) as well as for some observed cometary outbursts including those that occur at large heliocentric distances (e.g., West et al. 1991). We emphasize the importance of techniques such as radar reflection tomography and radiowave transmission tomography (e.g., Kofman et al. 1998) aboard cometary missions to determine the three dimensional structure of the nucleus in particular the extent of large scale voids.

keywords:

comets: general - comets: individual (LINEAR C/1999 S4) - structure 
To appear in Icarus (Received 10/19/2000; Accepted 05/21/2001)

\title{
A Model for the Breakup of Comet LINEAR (C/1999 S4)
}

\author{
Nalin H. Samarasinha \\ National Optical Astronomy Observatory ${ }^{1}$ \\ 950 N. Cherry Ave., Tucson, Arizona 85719 \\ nalinonoao.edu
}

\section{Introduction}

Comet LINEAR (C/1999 S4) was originally discovered by The Lincoln Near Earth Asteroid Research (LINEAR) program of the MIT Lincoln Lab on September 27, 1999 as an asteroidal object whereas the cometary nature of the object was noted by Durig et al. (1999) a few days later. The close perihelion distance $(0.77 \mathrm{AU})$ of the orbit and the prediction of a likely naked-eye visibility in July 2000 (Green 1999a, 1999b) attracted attention to this comet. As a result, it was monitored by different observers during the following months leading to the perihelion passage. The observations around the time of perihelion passage provided the details of the unexpected catastrophic breakup of comet LINEAR (C/1999 S4). These were the first such observations of a complete disruption of a cometary nucleus while the disruption was taking place (Kidger 2000, Licandro 2000, Weaver et al. 2001), providing an insight into the death of a comet, and in the process revealing clues about its structure.

In this paper, we propose a model to explain the catastrophic breakup of comet LINEAR (C/1999 S4). The model will additionally provide a viable mechanism which can explain some of the cometary outbursts for other comets - especially outbursts originating at large heliocentric distances (and also from the nightside).

\section{Comet LINEAR (C/1999 S4)}

Marsden et al. (1973) introduced radial and transverse nongravitational parameters, $A_{1}$ and $A_{2}$, with the nongravitational acceleration of the comet, $\mathbf{a}_{\mathbf{n g}}$, due to outgassing forces expressed by (neglecting the component normal to the orbital plane)

$$
\mathbf{a}_{\mathbf{n g}}=A_{1} g\left(r_{h}\right) \hat{\mathbf{r}}+A_{2} g\left(r_{h}\right) \hat{\mathbf{t}}
$$

\footnotetext{
${ }^{1}$ The National Optical Astronomy Observatory is operated by the Association of Universities for Research in Astronomy, Inc., under cooperative agreement with the National Science Foundation.
} 
where $r_{h}$ is the heliocentric distance and $g\left(r_{h}\right)$ describes the outgassing rate as a function of the heliocentric distance. $\hat{\mathbf{r}}$ is the instantaneous orbital unit vector in the outward radial direction while $\hat{\mathbf{t}}$ is the instantaneous transverse unit vector directed normal to $\hat{\mathbf{r}}$, in the orbital plane, and in the direction of the orbital motion.

Astrometry of comet LINEAR (C/1999 S4) revealed that it experienced comparatively large nongravitational accelerations when compared with those of other comets (cf. Marsden and Williams 1993); for LINEAR (C/1999 S4) $A_{1}=+9.5 \pm 0.23$ and $A_{2}=-2.11 \pm 0.09$ in units of $10^{-8} \mathrm{AU}_{\text {day }}{ }^{-1}$ (Marsden 2000). Even for the highly active comet 1P/Halley with localized outgassing (Keller et al 1986), the corresponding nongravitational parameters are smaller by more than an order of magnitude. As nongravitational accelerations are directly proportional to the net outgassing forces and inversely proportional to the nuclear mass (e.g., Yeomans and Chodas 1989, Samarasinha and Belton 1995), these large values of nongravitational parameters suggest a comparatively small nucleus. Our prior experience with modeling nongravitational forces (e.g., Samarasinha and Belton 1995, Samarasinha et al. 1996) indicates that the nuclear radius is of the order of one kilometer. We note that the exact number depends critically on the details of outgassing.

An independent lower limit to the nucleus radius can be derived from the total water production rate. Schleicher and Eberhardy (2000) derive a water production rate of $2.6 \times 10^{28}$ molecules s$~^{-1}$ (based on observations of $\mathrm{OH}$ ) on July 13,2000 when the heliocentric distance to the comet was 0.81 AU. Simple thermal models (e.g., Cowan and A'Hearn 1979, Julian et al. 2000) indicate this corresponds to a minimum nuclear radius of $\approx 0.7 \mathrm{~km}$ provided water was produced only by nucleus outgassing from the sunward hemisphere and the nucleus was not in an outburst during the observations.

Above arguments, while not conclusive, strongly favor that the nuclear radius of comet LINEAR (C/1999 S4) is of the order of one kilometer. Therefore, it is smaller than the recent bright comet, Hale-Bopp (C/1995 O1) (radius of 20-35 km; Weaver and Lamy 1997), and another extensively observed comet, $1 \mathrm{P} /$ Halley (effective radius of about $5 \mathrm{~km}$; Keller et al. 1986).

Weaver et al. (2001) estimate that the largest breakup fragments of LINEAR (C/1999 S4) are consistent with diameters of about $100 \mathrm{~m}$. This is consistent with the suggestion by Weidenschilling (1997) that 10-100 m size cometesimals are the likely building blocks of cometary nuclei based on his numerical simulations of growth of icy planetesimals in the outer solar system.

\section{Splitting/Breakup of Cometary Nuclei}

The splitting events, primarily the breaking away of cometary fragments from the nucleus are not uncommon. Based on the observations of 49 comets, Chen and Jewitt (1994) derive a lower limit of 1 comet splitting per comet per 100 years. There is extensive literature on the split comets including a review by Sekanina (1982). In a more recent paper, Sekanina (1997) revisits the subject and prefers a characterization based on whether the splitting occurred due 
to tidal interactions or not. Observational properties of split comets seem to depend on whether the splitting mechanism is of tidal origin or not. In particular, tidal disruption tends to produce fragments in a line, whereas other modes of disruption produce scattered fragments, as was the case for comet LINEAR (C/1999 S4).

There are a number of breakup mechanisms discussed in the literature for non-tidal splittings. These include rotational splitting (e.g., Sekanina 1982), including rotational splitting facilitated by evolution of the nucleus into a more elongated shape due to volatile loss (Hartmann and Tholen 1990), rotational splitting and/or gas pressure caused breakup facilitated by cracks of the nucleus which may occur either during phase transitions (e.g., Smoluchowski 1981, Jenniskens and Blake 1996) or via thermal stresses (e.g., Kührt 1984, Tambovtseva and Shestakova 1999).

Some of the mechanisms proposed to explain cometary outbursts can also lead to cometary breakups like the impact of an asteroidal object (e.g., Hughes 1991, Kadano 1999) and the exothermic polymerization of $\mathrm{HCN}$ (e.g., Rettig et al. 1992).

A model for explaining the breakup of the nucleus of comet LINEAR (C/1999 S4), unlike that for isolated splitting events, should explain why the whole nucleus underwent a catastrophic breakup. In other words, the model should provide a mechanism which can break the whole nucleus into multiple fragments abruptly consistent with the observations.

Rotational splitting is most likely to occur near the "equator" of the nucleus where the disruptive force due to rotation is the largest. It does not explain how the entire nucleus can be broken apart. A collision with an asteroidal object is most likely to occur near the ecliptic plane. However, the breakup of comet LINEAR (C/1999 S4) occurred close to perihelion when the comet was well away from the ecliptic plane. Furthermore, numerical studies by Asphaug et al. (1998) indicate energetic collision of a house size asteroidal object with a kilometer-sized rubble pile target is unlikely to disrupt the target. Finally, existing gas pressure based breakup mechanisms do not explain how the entire nucleus can be involved in a catastrophic breakup.

\section{Model}

We propose that the solid nucleus is made up of an aggregate of $10-100 \mathrm{~m}$ "cometesimals" (Weissman 1986, Weidenschilling 1997), and contains significant voids in the inter-cometesimal regions (see Fig 1 for a cartoon of the nucleus model). This network of inter-connected voids provides an efficient pathway for rapid propagation of super-volatile (i.e., species more volatile than water - dominated by $\mathrm{CO}$ and $\mathrm{CO}_{2}$ ) gasses over a wide regime of the nucleus causing gas pressure related stresses. These stresses, if sufficiently large, can eventually breakup the nucleus into the original "cometesimals" (i.e., its original building blocks).

The super-volatile gas production into inter-cometesimal voids can occur due to the inward propagation of the thermal wave as the comet comes closer to the sun. The exothermic phase 
transition of water ice from amorphous to crystalline may further facilitate the efficient inward propagation of the heat front (Prialnik and Bar-Nun 1987). Super-volatile gas release is associated with this phase transition (Prialnik and Bar-Nun 1987), due to restructuring of the water ice matrix caused by transitions between different forms of amorphous water ice occurring at lower temperatures (Jenniskens and Blake 1994), and possibly due to the exothermic polymerization of HCN (Rettig et al. 1992).

The propagation depth (skin depth), $\delta$, of the heat front is given by

$$
\delta=\left(\frac{K \tau}{\pi \rho C}\right)^{1 / 2}=\frac{I_{T}}{\rho C}\left(\frac{\tau}{\pi}\right)^{1 / 2}
$$

where $K, \rho, C$, and $I_{T}$ are thermal conductivity, density, specific heat, and thermal inertia of cometary ice respectively, while $\tau$ is the period of the heat wave. For a cometary-ice thermal inertia of $0.016 \mathrm{cal} \mathrm{cm} \mathrm{cm}^{-2} \mathrm{~s}^{-\frac{1}{2}} \mathrm{~K}^{-1}$ (which is based on model fits to water production rates in comet 1P/Halley; Julian et al. 2000), over the pre-perihelion leg, the propagation depth of the heat front for comet LINEAR (C/1999 S4) is of the order of $10 \mathrm{~m}$. The exothermic phase transitions described earlier would further enhance the propagation depth of the heat front. Comparable depths were derived by Prialnik and Bar-Nun (1987) for the crystallization front in case of a comet in the orbit of $1 \mathrm{P} /$ Halley. Likewise, Benkhoff and Huebner (1995) using an entirely different thermal model for the depth of the heat front for a comet in the orbit of $73 \mathrm{P} /$ Schwassmann-Wachmann 3 obtained similar results. Therefore, we argue that the thermal wave had sufficient time to propagate one cometesimal width (at least $10 \mathrm{~m}$ but likely more), in the case of comet LINEAR (C/1999 S4) by the time it broke up near perihelion.

The gravitational potential energy, $E_{g}$, of a constant density spherical nucleus with radius $R_{N}$ is given by

$$
E_{g}=\frac{3}{5} \frac{G M^{2}}{R_{N}}=\frac{16 \pi^{2}}{15} G \rho^{2} R_{N}^{5}
$$

where $M$ is the mass and $\rho$ is the bulk density of the nucleus while $G$ is the universal gravitational constant. Therefore, for a nucleus of $R_{N} \approx 1 \mathrm{~km}, E_{g} \approx 10^{18} \mathrm{ergs}$. Now, by assuming zero tensile strength against breakup, one can derive the minimum super-volatile gas mass required to completely disperse the nucleus due to gas pressure. The average kinetic energy for a mole of gas, $E_{k}$, is given by

$$
E_{k}=\frac{3}{2} R T
$$

where $T(\approx 100 \mathrm{~K})$ is the temperature and $R$ is the universal gas constant. From the above two equations, to completely disperse the nucleus due to super-volatile gas pressure, we derive a minimum gas mass of $10^{8}$ moles. This gas mass (when in the form of ice) would occupy onty a small fraction (of the order of $10^{-6}$ ) of the volume of a $R_{N} \approx 1 \mathrm{~km}$ nucleus and is consistent with our model. The solar constant (i.e., incident solar energy at $1 \mathrm{AU}$ ) is $1.4 \times 10^{6} \mathrm{ergs} \mathrm{s}^{-1} \mathrm{~cm}^{-2}$. Therefore, even if one assumes that the ultimate energy source for the breakup of the nucleus is solar energy 
(actually in the model, it acts only as the catalyst), a very small fraction of the total incident solar energy over the pre-perihelion leg can provide the necessary breakup energy.

Thermal models of the nucleus (e.g., Benkhoff and Huebner 1995, Capria et al. 1996, Enzian et al. 1999) which adopt a porous media for cometary ice find efficient gas diffusion through a series of unhindered tubal pores of radii typically of the order of $\mathrm{mm}$ or less. In those models where supervolatiles get depleted in the top most layers, they are produced at a depth and transported through pores in the porous media. Therefore, how can gas pressure buildup occur in the inter-cometesimal voids in our model? We suggest, that the process of collisional accretion leading to the formation of 10-100 m cometesimals (Weidenschilling 1997) did alter the structure of the accreting taget bodies. This structure alteration includes compaction and local melting at the collision sites - a sentiment expressed in Weissman (1986). Collision caused compaction of porous material is argued in Sirono and Greenberg (2000) and also seen in collision studies involving porous targets aimed at explaining large craters of the asteroid Mathilde (Housen et al. 1999). It is highly unlikely that cometesimals formed via a series of collisions could retain a set of unobstructed pores extending up to tens or hundreds of meters depth. When the porous media is modeled by a series of tubal pores, introduction of an effective scale length, $l$, for the "unobstructed pore length" is needed to account for the structure alterations discussed above. Unfortunately, to this author's knowledge, there are neither numerical nor laboratory studies on structure alterations applicable to icy cometesimals to derive a reliable number. While cometesimals can be porous, it is unlikely that their structure allows unhindered diffusion of gasses over tens or hundreds of meters. For example, for a depth of $10 \mathrm{~m}, l \approx 1 \mathrm{~m}$ would result in a decrease of more than four orders of magnitude (i.e., $e^{-10}$ ) for the effective number of available "unhindered" tubal pores. This facilitates gas pressure build up at high depths and has an effect similar to what a mantle does in the model of Möhlmann (1996).

Further evidence for our current insufficient understanding of the porous structure of cometary material and gas flow through it is apparent from the inability of the existing thermal models to accurately predict the observed CO production rate of comet Hale-Bopp (C/1999 O1) as a function of the heliocentric distance (Bockelee-Morvan and Rickman 1997). Therefore, further refinements to current thermal models, especially gas transport through cometary material, as well as exploring new mechanisms of super-volatile transport through the nucleus are required.

The other condition which prohibits a pressure build up in the inter-cometesimal voids is the presence of unobstructed large void outlets to the coma. Clearly, LINEAR (C/1999 S4) which broke up catastrophically (which was a rare event) was different from the majority of comets which do not undergo similar demises. We suggest what differentiates LINEAR (C/1999 S4) from other comets is lack of such void outlets to the coma thereby facilitating internal pressure build up. Blockage of surface outlets can occur due to reaccretion of "regolith" during the collisional accretion process (for example, see Leinhardt et al. 2000) and/or due to regolith redistribution associated with sublimation induced surface erosion - a surface process which has been proposed earlier (e.g., Sekanina 1991). For the purpose of calculating the rate of super-volatile gas effusion from any residual narrow openings to the coma at the blocked void outlets, let us define $A$ to be the sum of 
cross sections of all such openings. From kinetic theory, the relaxation time, $\tau_{\text {out }}$, of the confined gas is given by

$$
\tau_{\text {out }}=\frac{V}{A} \sqrt{\frac{2 \pi \mu}{R T}}
$$

where $V$ is the volume of the gas (assumed to be equal to the volume occupied by inter-cometesimal voids) and $\mu$ is the molar weight of gas.

In our model, the super-volatile gasses produced into voids can quickly spread throughout the network of inter-cometesimal voids. From the application of Bernoulli's principle and the ideal gas law, we derive

$$
v=\sqrt{\frac{2 R T}{\mu}}
$$

where $v$ is the gas propagation speed in the voids. Therefore, the propagation of gasses in intercometesimal voids occur on a timescale, $\tau_{\text {int }}$, given by

$$
\tau_{\text {int }} \approx \frac{2 R_{N}}{v}
$$

(i.e., of the order of 10-100 s). For the confinement of super-volatiles and the subsequent pressure buildup, $\tau_{\text {out }}>\tau_{\text {int }}$. i.e.,

$$
A \lesssim \frac{4 \sqrt{\pi^{3}}}{3} f R_{N}{ }^{2}
$$

where $f\left(=\frac{V}{\frac{4}{3} \pi R_{N}{ }^{3}} \ll 1\right)$ is the fractional volume of the inter-cometesimal voids. In the case of LINEAR (C/1999 S4), $A$ must be $\lesssim 10^{9} \mathrm{~cm}^{2}$ (i.e., less than $1 \%$ of the total surface area).

For the inter-cometesimal voids closest to the surface, the temperature of the cometesimal boundaries can be approximated by the amorphous to crystalline water ice transition temperature $(\approx 137 \mathrm{~K})$, but the cometesimal boundaries further inside the comet are likely to be much cooler, unless there is an interior energy source such as radiogenic heating under suitable conditions (e.g., Prialnik and Podolak 1995 and references therein). Consequently, condensation of super-volatile gasses can occur if $P_{i}>P_{i}^{s}(T)$, where $P_{i}$ is the gas pressure and $P_{i}^{s}(T)$ is the saturation gas pressure for a super-volatile species $i$. Therefore, the upper limit to the total gas pressure, $P_{u p p e r}$, can be expressed as

$$
P_{\text {upper }}=\sum_{i} P_{i}^{s}(T) \text {. }
$$

Breakup of the nucleus would occur if the disruptive forces (i.e., forces due to gas pressure and rotation) exceeds the binding forces due to self-gravity and large-scale tensile strength which holds the cometesimals together. We can mathematically express this as (see Appendix)

$$
P+\frac{2 \pi^{2} \rho R_{N}^{2} \cos ^{2} \lambda}{\tau_{N}^{2}} \geq \frac{2}{3} \pi G \rho^{2} R_{N}^{2}+\sigma
$$

where $P$ is the total gas pressure, $\tau_{N}$ is the rotation period of the nucleus, $\lambda$ is the latitude, and $\sigma$ is the large-scale tensile strength. By rearranging this equation and by substituting $P$ by $P_{u p p e r}$, 
we can derive an upper limit to the large-scale tensile strength.

$$
\sigma \leq P_{\text {upper }}+\frac{2 \pi^{2} \rho R_{N}^{2} \cos ^{2} \lambda}{\tau_{N}^{2}}-\frac{2}{3} \pi G \rho^{2} R_{N}^{2}
$$

For illustration purposes, by assuming that the gas pressure is dominated by the saturated gas pressure $P_{C O}^{s}$ of the most volatile and abundant super-volatile $\mathrm{CO}$ and neglecting the term due to rotation (even on the equator, rotation term can exceed the self-gravity term only if the rotation period is smaller than $\frac{3.3}{\sqrt{\rho}}$ hrs where $\rho$ is expressed in terms of $\mathrm{g} \mathrm{cm}^{-3}$; also Pravec and Harris 2000), we have

$$
\sigma_{\text {upper }}=P_{C O}^{s}-\frac{2}{3} \pi G \rho^{2} R_{N}^{2}
$$

Saturated gas pressure of $\mathrm{CO}$ can be approximated by

$$
P_{C O}^{s}=A_{C O} \exp \left(-\frac{B_{C O}}{T}\right)
$$

where $A_{C O}=1.2631 \times 10^{10}$ dyne $\mathrm{cm}^{-2}$ and $B_{C O}=764.16 \mathrm{~K}$ (Fanale and Salvail 1990). Fig 2 depicts the dependence of $\sigma_{\text {upper }}$ as a function of $P_{C O}^{s}$ and $R_{N}$. The binding effect due to self-gravity becomes relevant for a $R_{N}=1 \mathrm{~km}$ nucleus if $\sigma \lesssim 10^{3}$ dyne $\mathrm{cm}^{-2}$ while for a $R_{N}=10 \mathrm{~km}$ nucleus this holds true if $\sigma \lesssim 10^{5}$ dyne $\mathrm{cm}^{-2}$ (i.e., about $10^{-3}$ and $10^{-1}$ atmospheres respectively). Therefore, self-gravity can play an increasingly larger role in preserving larger nuclei.

\section{Discussion}

A major feature of the proposed model is the ability of super-volatile gasses to rapidly propagate within the inter-cometesimal voids. These natural voids created during the formation of a cometary nucleus in the solar nebula provide an efficient pathway for gas propagation. In the rubble pile model for cometary nuclei (Weissman 1986), the tensile strengths at which constituent "cometesimals" held together are lower than the tensile strengths of individual "cometesimals" themselves, thus providing a set of natural boundaries for breakup. Such a scenario is consistent with observations where the nucleus of comet LINEAR (C/1999 S4) broke into fragments of whose sizes are approximately in the same range as those predicted by Weidenschilling et al. (1997) for the basic building blocks of comets.

The proposed scenario raises the issue of why subsurface gas pressure broke up comet LINEAR (C/1999 S4) whereas not other comets. Inter-cometesimal voids lacking sufficiently large unobstructed outlets to the coma which facilitates internal gas pressure build up, as we suggest for LINEAR (C/1999 S4), differentiate it from the majority of comets which do not undergo similar catastrophic breakups. In addition, small cometary nuclei such as comet LINEAR (C/1999 S4) are more prone to such complete catastrophic breakups due to gas pressure driven mechanisms than larger nuclei. Larger cometary nuclei are more stable because of the combined effects of (a) the ability of the larger self-gravity to hold the nucleus together (which scales as $R_{N}{ }^{2}$; see previous 
section), (b) a larger ratio of volume of voids to rate of super-volatile production (which scales as $R_{N}$ ), and (c) the increased likelyhood of voids having a large unobstructed outlet to the coma (which scales as $R_{N}^{2}$ ).

In a different context, large scale subsurface propagation of gasses was previously proposed by Möhlmann (1996), where he suggests that the active regions of water outgassing on cometary nuclei are simply local "source sites" of accumulated subsurface flows from distant regions in the nucleus, in contrast to water sublimation occurring predominantly at the surface in the conventional thermal models. Model results from a scenario as proposed by Möhlmann (1996) need to be compared with observations, in particular the water production rate and its variations with heliocentric distance and nucleus rotation.

If the inter-cometesimal voids have an unobstructed outlet to the coma, this same physical mechanism can be responsible for some of the observed cometary outbursts, such as those observed at large heliocentric distances. A likely example is the $14 \mathrm{AU}$ outburst of comet $1 \mathrm{P} / \mathrm{Halley}$ (West et al. 1991). Observationally verifying whether an outburst is related to a super-volatile gas pressure driven mechanism is possible as there should be enhanced abundances of species more volatile than water during an outburst. In fact, such an association has been made by Feldman et al. (1986) in the case of comet $1 \mathrm{P} /$ Halley.

The issues raised in this paper point to the necessity of detailed numerical and laboratory studies of porous structure of collisionally formed "cometesimals" as well as detailed thermal models of the nucleus based on inputs from such studies in the future.

Finally, we wish to point out the importance of effective three dimensional structure deterministic techniques such as radar reflection tomography (M. Belton, private communication) and radiowave transmission tomography (e.g., CONSERT experiment aboard Rosetta; see Kofman et al. 1998) in future cometary missions. Such determinations would not only tell us the three dimensional structure of cometary nuclei, but also be useful to assess the likely role of subsurface structure in cometary activity and breakup events.

\section{Appendix}

The inequality for the non-tidal breakup of a nucleus can be expressed as $P+F_{R} \geq F_{G}+\sigma$ where $P$ is the total interior gas pressure, $F_{R}$ is the disruptive force due to rotation per unit area, $F_{G}$ is the binding force due to self-gravity per unit area, and $\sigma$ is the large-scale tensile strength holding cometesimals together. We derive $F_{R}$ and $F_{G}$ for a spherical nucleus.

\section{Derivation of $F_{R}$ :}

Consider a ring of cometary material at latitude $\lambda$ having width $r d \lambda$ and height $d r$. The disruptive force due to rotation is given by $\frac{m_{r i n g} V^{2}}{r}=\frac{(2 \pi r \rho \cos \lambda r d \lambda d r)\left(2 \pi r \cos \lambda / \tau_{N}\right)^{2}}{r}$ where $m_{\text {ring }}$ is the mass of the ring, $V$ is the speed of rotation, $r$ is the distance from the center of the nucleus, $\rho$ is the bulk density of the nucleus, and $\tau_{N}$ is the rotation period. As this force acts upon an 
area of $2 \pi r \cos \lambda r d \lambda$, the force per unit area is given by $\frac{4 \pi^{2} \rho \cos ^{2} \lambda r d r}{\tau_{N}^{2}}$. By integrating we find $F_{R}=\frac{2 \pi^{2} \rho R_{N}{ }^{2} \cos ^{2} \lambda}{\tau_{N}^{2}}$ across the entire nuclear radius $R_{N}$. This expression for $F_{R}$ is consistent with the one cited in Sekanina (1982) for $\lambda=0$.

\section{Derivation of $F_{G}$ :}

Consider a spherical shell of cometary material having thickness $d r$. The gravitational force on the shell is given by $\frac{G m_{\text {int }} m_{\text {shell }}}{r^{2}}=\frac{G\left(\frac{4}{3} \pi \rho r^{3}\right)\left(4 \pi \rho r^{2} d r\right)}{r^{2}}$ where $G$ is the universal gravitational constant, $m_{\text {int }}$ is mass interior to the shell, and $m_{\text {shell }}$ is mass of the shell. Since this force is acted upon an area of $4 \pi r^{2}$, the force per unit area is given by $\frac{4}{3} \pi G \rho^{2} r d r$. By integrating we get $F_{G}=\frac{2}{3} \pi G \rho^{2} R_{N}{ }^{2}$ across the entire nuclear radius.

\section{Acknowledgements}

I thank Drs. M.J.S. Belton and B.E.A. Mueller for their comments on an early version of this paper. I appreciate the constructive suggestions from the anonymous referees which helped in improving the paper. This work was supported by a grant from NASA.

\section{References}

Asphaug, E., S.J. Ostro, R.S. Hudson, D.J. Scheeres, and W. Benz 1998. Disruption of kilometresized asteroids by energetic collisions. Nature 393, 437-440.

Benkhoff, J., and W.F. Huebner 1995. Influence of the vapor flux on temperature, density, and abundance distributions in a multicomponent, porous, icy body. Icarus 114, 348-354.

Bockelee-Morvan, D., and H. Rickman 1997. C/1995 O1 (Hale-Bopp): Gas production curves and their interpretation. Earth, Moon, and Planets 79, 55-77.

Capria, M.T., F. Capaccioni, A. Coradini, M.C. De Sanctis, S. Espinasse, C. Federico, R. Orosei, and M. Salomone 1996. A P/wirtanen evolution model. Planet. Space Sci. 44, 987-1000.

Chen, J., and D. Jewitt 1994. On the rate at which comets split. Icarus 108, 265-271.

Cowan, J.J. and M.F. A'Hearn 1979. Vaporization of cometary nuclei: light curves and lifetimes. Moon Planets 21, 155-171.

Durig, D., J. Ticha, and M. Tichy 1999. IAU Circular 7267.

Enzian, A., J. Klinger, G. Schwehm, and P.R. Weissman 1999. Temperature and gas production distributions on the surface of a spherical model comet nucleus in the orbit of $46 \mathrm{P} / \mathrm{Wirtanen}$. Icarus 138, 74-84. 
Fanale, F.P., and J.R. Salvail 1990. The influence of CO ice on the activity and near-surface differentiation of comet nuclei. Icarus 84, 403-413.

Feldman, P.D., H. Weaver, M.F. A'Hearn, M.C. Festou, and L.A. McFadden 1986. Is $\mathrm{CO}_{2}$ responsible for the outbursts of comet Halley? Nature 324, 433-436.

Green, D.W.E. 1999a. IAU Circular 7267.

Green, D.W.E. 1999b. IAU Circular 7285.

Hartmann, W.K., and D.J. Tholen 1990. Comet nuclei and Trojan asteroids - A new link and a possible mechanism for comet splittings. Icarus 86, 448-454.

Housen, K.R., K.A. Holsapple, and M.E. Voss 1999. Compaction as the origin of the unusual craters on the asteroid Mathilde. Nature 402, 155-157.

Hughes, D.W. 1991. Comet Halley's outburst, Mon. Not. R. Astron. Soc. 251, 26p-29p.

Jenniskens, P., and D.F. Blake 1994. Structural transitions in amorphous water ice and astrophysical implications. Science 265, 753-756.

Jenniskens, P., and D.F. Blake 1996. A mechanism for forming deep cracks in comets. Planet. Space Sci. 44, 711-713.

Julian, W.H., N.H. Samarasinha, and M.J.S. Belton 2000. Thermal structure of cometary active regions: Comet 1P/Halley. Icarus 144, 160-171.

Kadano, T., 1999. Hypervelocity impact into low density material and cometary outburst. Planet. Space Sci. 47, 305-318.

Keller, H.U., and 17 co-authors 1986 . First Halley multicolour camera imaging results from Giotto. Nature 321, 320-326.

Kidger, M. 2000. IAU Circular 7467.

Kofman, W., and 20 co-authors 1998 . Comet nucleus sounding experiment by radiowave transmission. Adv. Spcae Res. 21, 1589-1598.

Kührt, E. 1984. Temperature profiles and thermal stresses in cometary nuclei. Icarus 60, 512-521.

Leinhardt, Z.M., D.C. Richardson, and T. Quinn 2000. Direct N-body simulations of rubble pile collisions. Icarus 146, 133-151.

Licandro, J., G. Tessicini, I. Perez, and S. Hidalgo 2000. IAU Circular 7468.

Marsden, B.G. 2000. Minor Planet Electronic Circular 2000-007. 
Marsden, B.G., Z. Sekanina, and D.K. Yeomans 1973. Comets and nongravitational forces V. Astron. J. 78, 211-225.

Marsden, B.G., and G.V. Williams 1993. Catalogue of Cometary Orbits 1993, IAU Central Bureau for Astronomical Telegrams, SAO, Cambridge, Mass.

Mekler, Y., D. Prialnik, and M. Podolak 1990. Evaporation from a porous cometary nucleus. Astrophys. J. 356, 682-686.

Möhlmann, D. 1996. Cometary activity and nucleus modelling: A new approach. Planet. Space Sci. 44, 541-546.

Pravec, P., and A.W. Harris 2000. Fast and slow rotation of asteroids. Icarus 148, 12-20.

Prialnik, D., and A. Bar-Nun 1987. On the evolution and activity of cometary nuclei. Astrophys. J. 313, 893-905.

Prialnik, D., and M. Podolak 1995. Radioactive heating of porous comet nuclei. Icarus 117, 420-430.

Rettig, T.W., S.C. Tegler, D.J. Pasto, and M.J. Mumma 1992. Comet outbursts and polymers of HCN. Astrophys. J. 398, 293-298.

Samarasinha, N.H., and M.J.S. Belton 1995. Long-term evolution of rotational states and nongravitational effects for Halley-like cometary nuclei. Icarus 116, 340-358.

Samarasinha, N.H., B.E.A. Mueller, and M.J.S. Belton 1996. Comments on the rotational state and non-gravitational forces of comet 46P/Wirtanen. Planet. Space Sci. 44, 275-281.

Schleicher, D., and C. Eberhardy 2000. IAU Circular $\mathbf{7 4 5 5}$.

Sirono, S., and J.M. Greenberg 2000. Do cometesimal collisions lead to bound rubble piles or to aggregates held together by gravity? Icarus 145, 230-238.

Sekanina, Z. 1982. The problem of split comets in review. In Comets (L.L Wilkening, Ed.), pp 251-287. Univ. of Arizona Press, Tucson, Arizona.

Sekanina, Z. 1991. Cometary activity, discrete outgassing, areas, and dust-jet formation. In Comets in the Post-Halley Era, Vol. 2 (R.L. Newburn, Jr., M. Neugebauer, and J. Rahe, Eds.), pp 769-823. Kluwer Academic Publishers, The Netherlands.

Sekanina, Z. 1997. The problem of split comets revisited. Astron. Astrophys. 318, L5-L8.

Smoluchowski, R., 1981. Amorphous ice and the behavior of cometary nuclei. Astrophys. J. 244, L31-L34.

Tambovtseva, L.V., and L.I. Shestakova 1999. Cometary splitting due to thermal stresses. Planet. Space Sci. 47, 319-326. 
Weaver, H,A., and P.L. Lamy 1997. Estimating the size of Hale-Bopp's nucleus. Earth, Moon, and Planets 79, 17-33.

Weaver, H.A., and 20 colleagues 2001. HST and VLT investigations of the fragments of comet C/1999 S4 (LINEAR). Science 292, 1329-1333.

Weidenschilling, S.J. 1997. The origin of comets in the solar nebula: A unified model. Icarus 127, 290-306.

Weissman, P.R. 1986. Are cometary nuclei primodial rubble piles? Nature 320, 242-244.

West, R.M., O. Hainaut, and A. Smette 1991. Post-perihelion observations of P/Halley III. An outburst at $r=14.3$ AU. Astron. Astrophys. 246, L77-L80.

Yeomans, D.K., and P.W. Chodas 1989. An asymmetric outgassing model for cometary nongravitational accelerations. Astron. J. 98, 1083-1093. 
Fig. 1.- A cartoon showing a 2-D cross section of the 3-D nucleus model containing intercometesimal voids and individual cometesimals. In the cartoon, the cometesimals are shown in light gray while arrows denote regions where outgassing can occur. Outgassing of volatiles (dominated by water) can occur from non-mantled cometesimal surfaces. In addition, super-volatiles can escape from void outlets not covered by regolith/debris. In the case of comet Linear (C/1999 S4), we suggest that the regolith/debris obstructs all the void outlets, thereby facilitating internal pressure buildup. Note that the cartoon is not drawn to scale (fог example, the number of cometesimals is underrepresented and small scale features are exaggerated). Therefore, we caution the reader not to overinterpret the cartoon.

Fig. 2.- Dependance of $\sigma_{\text {upper }}$ as a function of $P_{C O}^{s}$ (as well as $T$ ) and $R_{N}$. Nuclear bulk density of $0.5 \mathrm{~g} \mathrm{~cm}^{-3}$ is assumed in calculations. For a given nuclear radius, unless the tensile strength is sufficiently large, the disruptive forces can break/split the nucleus as self-gravity cannot hold the nucleus together. This critical tensile strength increases with nuclear radius. 


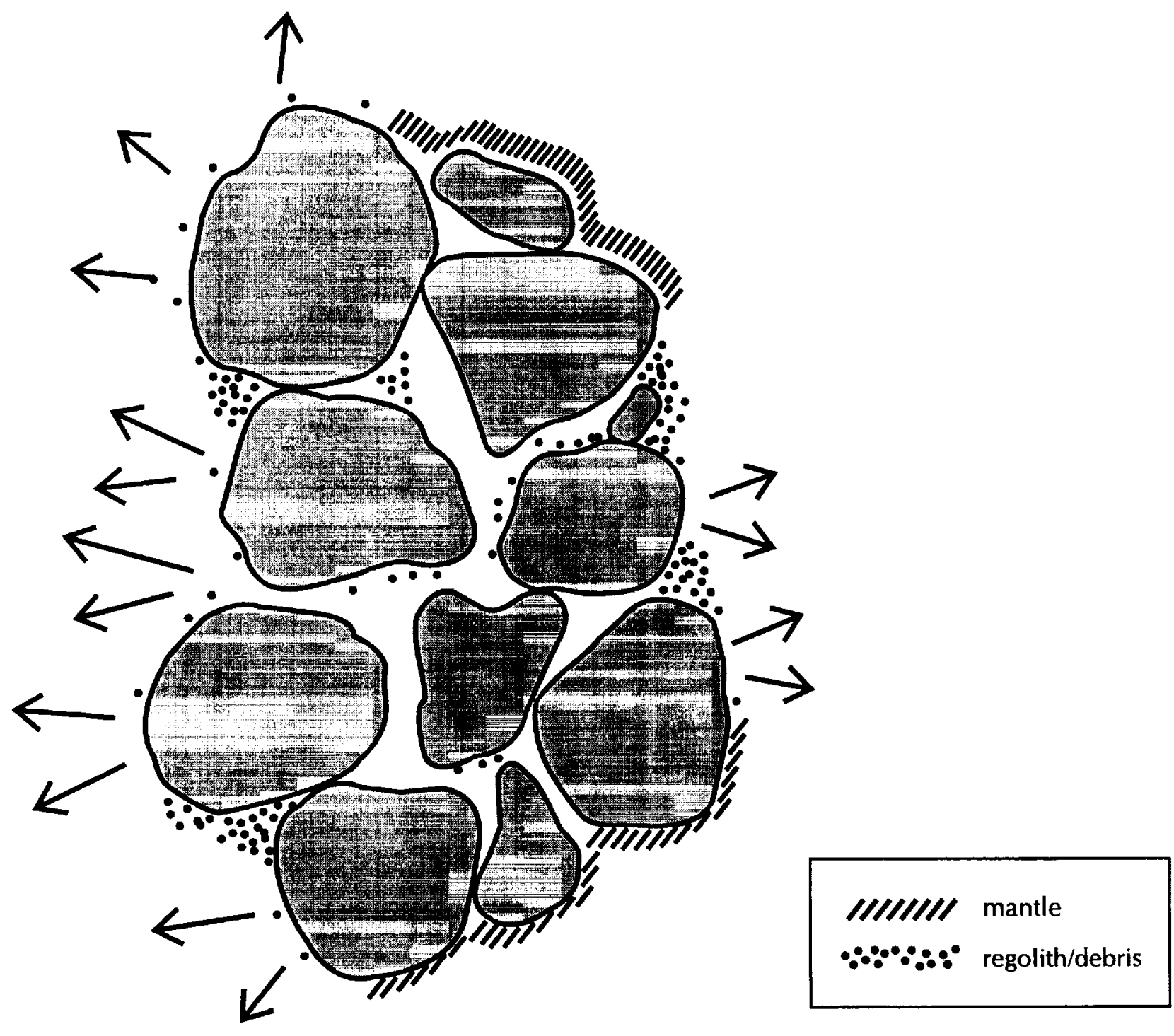

Fig. I 


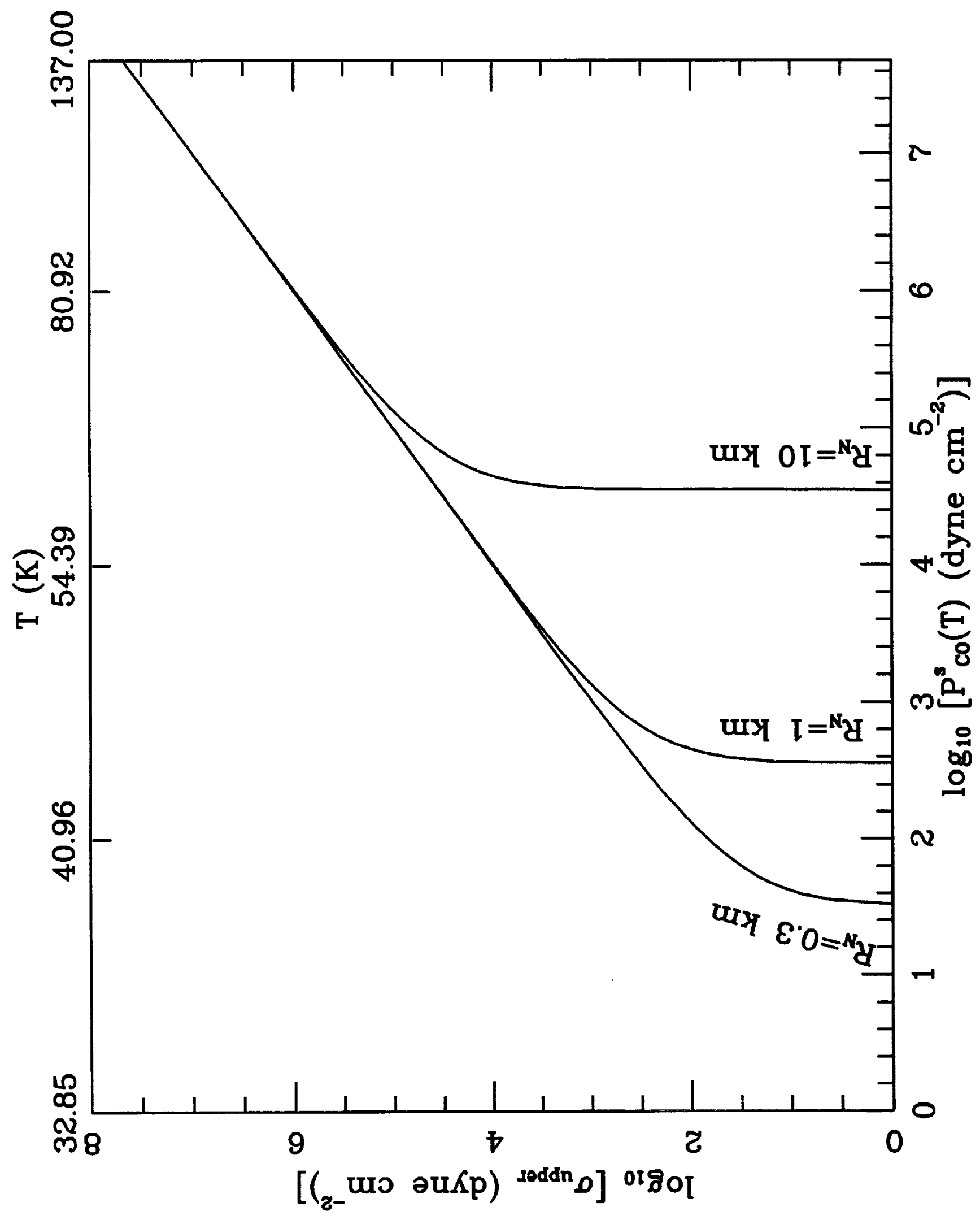




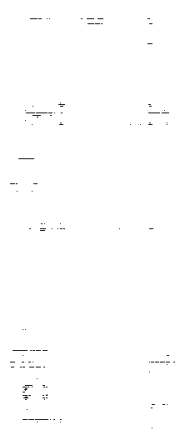

